\title{
Electromagnetic forces in cage induction motors with rotor eccentricity
}

\author{
A. Tenhunen, T. Benedetti, T. P. Holopainen*, A. Arkkio \\ Laboratory of Electromechanics \\ Helsinki University of Technology \\ P.O. Box 3000, FIN-02015 HUT, Finland \\ Asmo.Tenhunen@hut.fi \\ *VTT Industrial Systems \\ Technical Research Centre of Finland \\ P.O. Box 13022, FIN-02044 VTT, Finland \\ Timo.Holopainen@vtt.fi
}

\begin{abstract}
The paper deals with the electromagnetic forces in induction machines when the rotor is performing eccentric motion with respect to the stator. The studied eccentric motions of the rigid cage rotor are cylindrical circular whirling motion, symmetric conical whirling motion and the combination of these two basic modes of eccentric motions.

The multi-slice, time stepping finite element analysis is used for solving the magnetic field, and the forces are calculated from the air gap by a method based on the principle of virtual work. The forces are measured for a test motor equipped with active magnetic bearings. The active magnetic bearings are used to generate the eccentric rotor motions and also to measure the electromagnetic forces. The measured and calculated forces show relatively good agreement. The results show that superposition is valid when determining forces. This means that the calculated forces of different motions can be combined and the result is the force of the combined motion.
\end{abstract}

\section{INTRODUCTION}

The electromagnetic force acts between the rotor and stator when the rotor is not perfectly concentric and aligned with the stator. In this paper, the forces are studied during different kind of eccentric motions of the rigid rotor. The eccentric motions of the rigid rotor are divided into the two basic modes, cylindrical whirling motion and symmetric conical whirling motion. The cylindrical whirling motion of the rotor means that the rotor remains aligned with the stator but the geometrical centerline of the rotor travels around the geometrical centerline of the stator in a circular orbit with a certain frequency known as the whirling frequency, and with a certain radius, known as the whirling radius. The symmetric conical whirling motion of the rotor means that the whirling radius at each end of the rotor is equal but in opposite directions and the whirling frequency remains constant. These modes of rotor motions and a combination of them are illustrated in Fig. 1.

The eccentric motions of the rotor can be almost whatever motions, in which the centerline of the rotor deviates from the centerline of the stator, at least, at some time instant. To simplify the task, the present work focuses on the whirling motion of the rotor and on the first harmonic force component i.e. the force at the whirling frequency. Also, for simplicity, only the rigid rotor motions are considered i.e. the bending of the rotor is neglected.

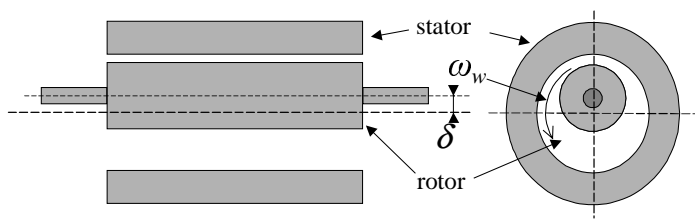

a) Cylindrical whirling motion

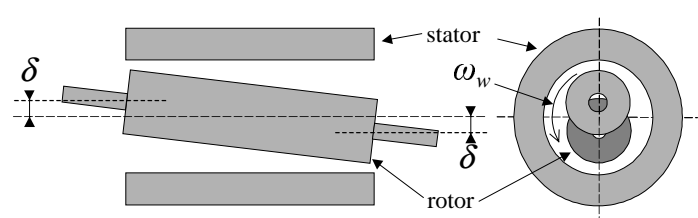

b) Symmetric conical whirling motion

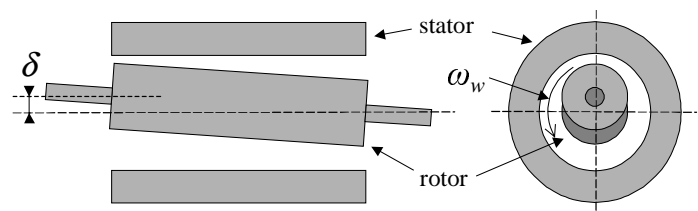

c) A combination of the two whirling motions

Fig. 1. Studied types of rigid rotor motions. In figures, $\delta$ is the whirling radius of the rotor.

Conventionally the electromagnetic forces acting between the rotor and stator have been studied analytically [1-3]. Most of these studies focused on two special cases of the whirling motion, i.e. static and dynamic eccentricity. Früchtenicht et al. [4] derived equations for the forces in induction machines in cylindrical whirling motion. Arkkio et al. [5] studied the forces in whirling motion by finite element analysis and verified their results by measurements. They also presented a low order force model, which presents the forces as a function of whirling frequency, assuming that the force is a linear function of the displacement. Based on the assumption of the spatial linearity, Tenhunen et al. [6] calculated the electromagnetic forces from the impulse response of the rotor displacement.

The references given above focus on the type of eccentricity in which the rotor is aligned with the stator. Dorrell [7] developed analytical tools to study the forces for the static and dynamic eccentricity of the conical motion. The static and dynamic conical motions of the rotor are studied numerically in [8], in which the effect of parallel branches on the forces is also considered.

The aim of this study is to investigate the superposition for the forces when the rotor is performing combined cylindrical 
and conical whirling motion and the whirling frequencies of these two motions are not equal. This means that the forces calculated for the cylindrical and conical motion can be superposed to obtain the force for the combined motion.

\section{METHODS OF ANALYSIS}

\section{A. Numerical calculation}

In this study, the calculation of the operating characteristics of the induction motor is based on time-stepping, multislice, finite element analysis of the magnetic field. The details of the method are given in [9]. In the multi-slice modeling, the motor is divided into slices cut by planes perpendicular to the stator axis. The slices are connected together by forcing the currents in the stator windings and rotor cage to be continuous from slice to slice. The magnetic field in the core region of the slice of the motor is assumed to be twodimensional, and the two-dimensional field equation is discretized by the finite element method. The effects of end region fields are taken into account approximately by constant end-winding impedances in the circuit equations of the windings. The field equation and the circuit equations are solved together as a system of equations. The timedependence of the field is modeled by Crank-Nicholson method. The magnetic field, the currents and the potential differences of the windings are obtained in the solution of the coupled field and circuit equations.

The method based on the principle of virtual work presented by Coulomb [10] is used to compute the electromagnetic forces. In the two-dimensional formulation, the force is obtained as a surface integral over the finite elements in the air gap.

The motion of the rotor is obtained by changing the finite element mesh in the air gap. The cylindrical whirling motion of the rotor is modeled using one slice, which is equivalent with conventional finite element analysis. The analysis of the conical motions is done using three slices with equal length in the multi-slice model. We used second-order, isoparametric, triangular elements and a typical finite element mesh for one cross section of the motor contained about 10000 nodes.

The impulse method in the finite element analysis is utilized in the calculation of the force between the stator and the rotor for the basic modes of the whirling motion. The details of the impulse method are presented in reference [6]. The basic idea of the impulse method is to move the rotor from its central position for a short period of time to one direction, fixed in the stator co-ordinate system. This displacement excitation disturbs the flux density distribution, and by doing this, produces forces between the rotor and stator. Using spectral analysis techniques, the frequency response function of the force is determined from the excitation and response signals.

The length of the rectangular displacement pulse in the simulation was $0.01 \mathrm{~s}$. The amplitude of the static pulse was $11 \%$ of the air gap length for the whirling and the conical modes and $22 \%$ for the combined motion. Total simulation time was $1.0 \mathrm{~s}$ with constant time-step of $0.05 \mathrm{~ms}$. To increase the spectral resolution, the sample size was extended to be $2 \mathrm{~s}$ by adding zeros to the end of the sample. This leads to frequency resolution of $0.5 \mathrm{~Hz}$. The discrete excitation and force signals were transformed to the frequency domain by the fast Fourier transform without filtering or windowing. The number of sample points was 8192 .

Based on the spatial linearity of the force [11], the frequency response defines the forces for a specific whirling radius for the studied whirling frequency range.

To ensure the calculated results for the combined motion, the forces were also calculated by conventional way, called here forced whirling method. In the time stepping finite element analysis, the center point of the rotor at each slice was forced to move along the orbit, corresponding to the combined eccentric motion $[5,8]$.

To be able to compare the calculated and measured results in the conical motions, the calculated forces of the slices are presented by the moment of force about the center point of the rotor (Fig. 2) via force distribution.

Supposing that the force distribution has the spatial linearity property [11], the calculated slice forces can be presented by a force distribution $F_{\mathrm{d}}(z)=g z+k$ in which $z$ is the axial position in the shaft and $k^{\prime}$ and $g$ ' are coefficients (Fig. 2). The coefficient $g$ is related to the force distribution due to the symmetrical conical motion by angle $\theta$ and the coefficient $k$ is related to the force distribution caused by the cylindrical motion. The force distribution can be defined from the calculated forces acting on slice $n$

$$
F_{n}=\int_{a}^{b} F_{\mathrm{d}}(z) d z=\int_{a}^{b} g z+k d z
$$

where $F_{n}$ is the force acting on slice $n$ and $b$ and $a$ are the end position coordinates of the slice.

The coefficients $k^{\prime}$ and $g$ ' are solved using (1) and calculated forces of the slices. The moment of the force $M$ about the center point of the rotor is then defined as

$$
M=\int_{-1 / 2}^{1 / 2} F_{d}(z) z d z
$$

in which $l$ is the length of the rotor.

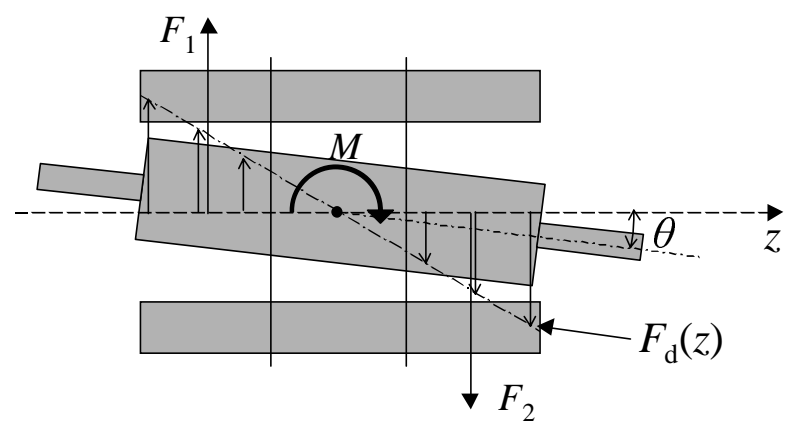

Fig. 2. The forces $F_{1}$ and $F_{2}$ of the slices, force distribution $F_{\mathrm{d}}(z)$ and moment of the force $M$ about the center point of the rotor in conical mode motion. 


\section{B. Measurements}

The test machine is a $15 \mathrm{~kW}$ four-pole cage induction motor. The main parameters of the motor are given in Table I. The test motor was equipped with radial magnetic bearings to measure the forces and to generate the eccentric motions of the rotor. Only the radial bearings were installed, because the electrical machine itself acts as an axial bearing. The radial bearings were ordinary eight-pole heteropolar bearings with bias-current linearization.

The calibration of the active magnetic bearings and the measurements were done following the procedure presented in [5]. The axial rotor geometry of the test motor is shown in Fig. 3. To get the symmetric conical motion in the measurement, one extra calibration was done. The trajectory of the rotor was determined in such a way that the total force acting on the rotor was zero (i.e. $F_{1}=-F_{2}$ in Fig. 3 ). Then, the center point of the rotor is concentric and the conical motion causes only the moment acting on the center point of the rotor. In reality, we were not able to balance bearing forces perfectly. This can be seen from the results of measurements.

The measured total force $F$ acting on the rotor during the eccentric motion and the moment $M$ acting in its center are measured through the forces $F_{1}$ and $F_{2}$ acting in the magnetic bearings, using the basic laws of mechanical equilibrium (see Fig. 2)

$$
\begin{aligned}
& F=F_{1}+F_{2} \\
& M=F_{1} l_{1}+F_{2} l_{2}
\end{aligned}
$$

where $F_{1}$ and $F_{2}$ are the measured forces in the center points of the magnetic bearings and $l_{1}=0.2725 \mathrm{~m}$ and $l_{2}=0.2475 \mathrm{~m}$ are the distances of these two points from the center point of the rotor.

TABLE I

MAIN PARAMETERS OF THE TEST MOTOR.

\begin{tabular}{|l|c|}
\hline Parameter & \\
\hline Number of poles & 4 \\
\hline Number of phases & 3 \\
\hline Number of parallel paths & 1 \\
\hline Outer diameter of stator [mm] & 235 \\
\hline Core length [mm] & 195 \\
\hline Inner diameter of stator [mm] & 145 \\
\hline Airgap length [mm] & 0.45 \\
\hline Number of stator slots & 36 \\
\hline Number of rotor slots & 34 \\
\hline Connection & Delta \\
\hline Skew & 0 \\
\hline Rated voltage [V] & 380 \\
\hline Rated frequency [Hz] & 50 \\
\hline Rated current [A] & 28 \\
\hline Rated power [kW] & 15 \\
\hline
\end{tabular}

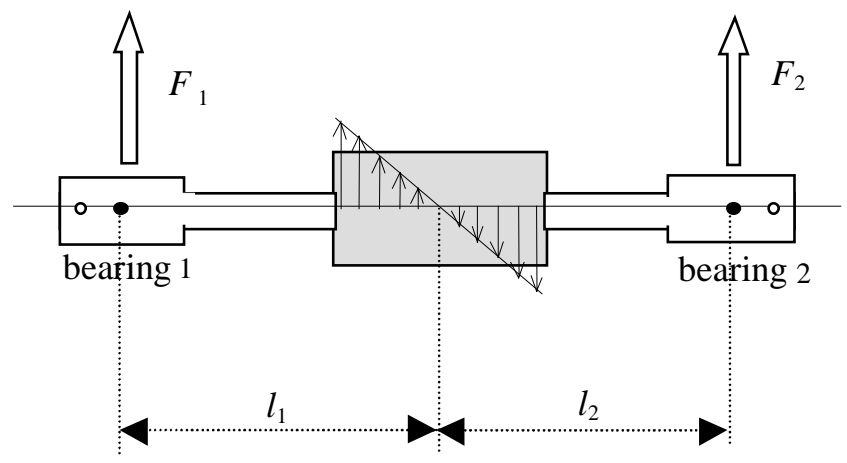

Fig. 3. Axial rotor geometry of the test machine - centers of bearing magnets o centers of position sensors $\rightarrow$ magnetic forces acting on the rotor $\Rightarrow$ forces on the magnetic bearings

The effect of inertia forces was eliminated by subtracting the forces obtained in similar conditions without voltage supply from the forces obtained with voltage supply.

The motor is running at no load and supplied by sinusoidal $230 \mathrm{~V}$ three-phase voltage. It was necessary to reduce the voltage level from the rated $380 \mathrm{~V}$ to keep the magnetic bearings at the linear range of operation to guarantee accurate force measurements. Because of the small clearance available between the ends of the shaft and the touchdown bearings near to the magnetic bearings, a whirling radius $\delta=20 \mu \mathrm{m}$ (i.e. about $4.4 \%$ of the air gap) was chosen for most of the measurements. For the cylindrical whirling motion, the touchdown bearings did not limit the whirling radius.

\section{RESULTS}

\section{A. Computed Results}

Fig. 4 shows the calculated trace of the force vector during the cylindrical whirling motion with whirling frequency 25 Hz. The simulation has not reached steady state because the amplitude of the force is still increasing a bit. The motor is running at no load and supplied by sinusoidal $230 \mathrm{~V}$ voltage. According to the analytical theory [4], the basic component of the force vector is rotating at the whirling frequency. The slotting causes small fluctuations in the force, but the trace is quite close to a circle. If the force vector is divided into a radial component in the direction of the shortest air gap and a tangential component perpendicular to the radial one, the components are almost independent of time [5].

The net force between the rotor and stator and the moment acting in the center point of the rotor are computed for cylindrical and symmetric conical whirling motion and for the combination of them as a function of whirling frequency using impulse method. 


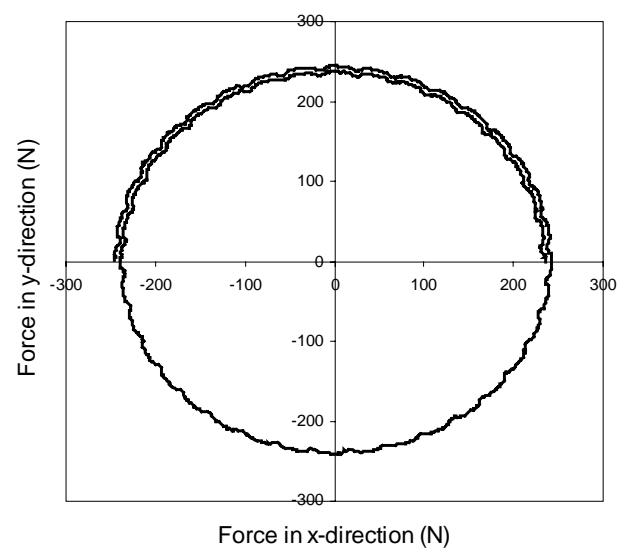

Fig. 4. Typical trace of the force vector obtained in the time stepping simulations of an unloaded motor supplied by 230 voltage.

In the cylindrical whirling motion, the whirling radius was $20 \mu \mathrm{m}$. The motor was running at no load and supplied by sinusoidal $230 \mathrm{~V}$ voltage. The total force as a function of whirling frequency is shown in Fig. 5 by a continuous line. It also shows the net force of the combined eccentric motion in which one end of the rotor is concentric and the other one is performing whirling motion with $40 \mu \mathrm{m}$ whirling radius. This motion presents combination of the cylindrical and the symmetric conical whirling motion with equal whirling frequencies and radii of $20 \mu \mathrm{m}$.

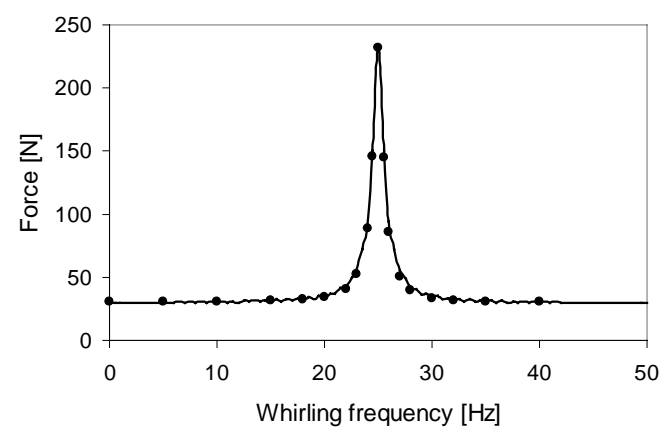

a)

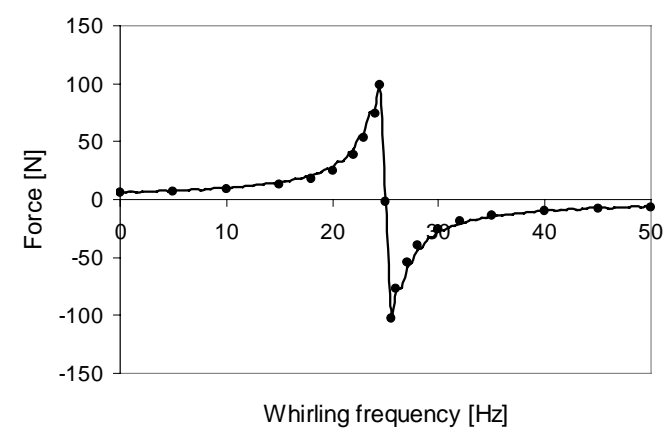

b)

Fig. 5. The total force as a function of the whirling frequency.

a) Radial component

b) Tangential component

- $\quad$ Combined cylindrical and conical motion
The calculated net force acting between the rotor and stator was zero in the symmetric conical motion. For both, the symmetric conical and combined motion, the calculated moment $M$ acting on the center point of the rotor due to the conical motion was constant $8.46 \mathrm{Nm}$ at the studied whirling frequency range.

Since now, we have studied the combined motion for which the whirling frequencies of the basic modes are equal. The net force and moment were also calculated for other combined motions, in which the whirling frequency $f_{w}$ of the cylindrical or symmetric conical motion were kept constant ( $f_{w}=0 \mathrm{~Hz}$ i.e. static rotor displacement) and the force and the moment of the other component of the motion were calculated as a function of whirling frequency. The static displacement causes an approximately constant force component, which shifts the rotating force vector away from the origo in Fig. 4. The trace of the rotating force vector is similar as in Fig 4. The analyzed cases are: 1) conical motion around an axis with static cylindrical displacement, 2) cylindrical whirling motion around the axis with symmetric conical displacement from the stator axis and 3) cylindrical whirling motion around the axis with static cylindrical displacement.

As a result, the forces versus whirling frequency in case 2) and 3) are almost the same as presented in Fig. 5, except in case 3 ) at the whirling frequency $0 \mathrm{~Hz}$ when the force is double compared to Fig. 5. In this point, the combined motion corresponds to a static displacement with a whirling radius of $40 \mu \mathrm{m}$. The moment in case 1) is constant $8.46 \mathrm{Nm}$ at the studied whirling frequency range and in case 2) is constant $8.46 \mathrm{Nm}$ at whirling frequency $0 \mathrm{~Hz}$.

\section{B. Measured results}

The electromagnetic force and moment were measured for the same kind of eccentric motions of the rotor as calculated following the procedure presented in the previous section.

At first, the measurements were done for the cylindrical, symmetric conical and combined motion, in which the whirling frequencies of the cylindrical and conical motions were the same. The results of this combined motion are compared with the sum of results of the cylindrical and conical motions in Fig. 6. The forces seem to be almost equal, and close to the calculated ones shown in Fig. 5. In the moment $M$, small differences occur. One reason for this is that the force components, which cause the moment $M$, acting on the magnetic bearings were quite small. Accurate definition of small force components was quite hard task in the used measurement set-up in which the resolution was about 0.5 $\mathrm{Nm}$.

In the cylindrical motion, a small component of moment $(<0.5 \mathrm{Nm})$ and in the symmetric conical motion, a small component of net force $(<2 \mathrm{~N})$ always occurs, maybe because the rotor and stator were not perfectly cylindrical. That is the reason why the sum of results is used when comparing the measured results. 


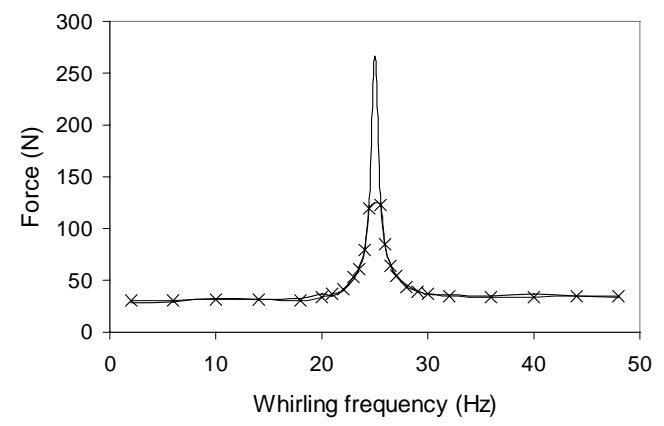

a)

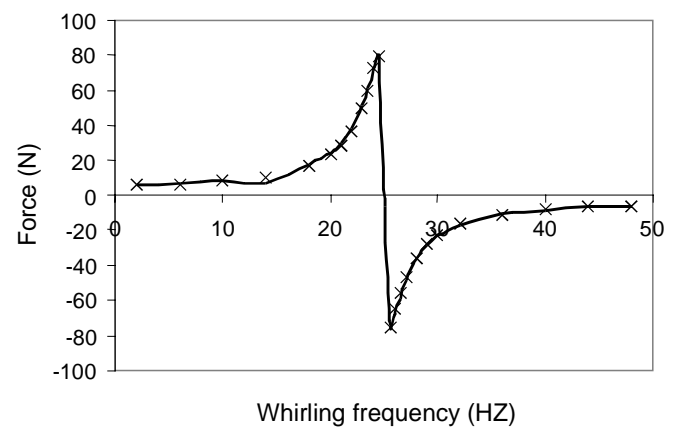

b)

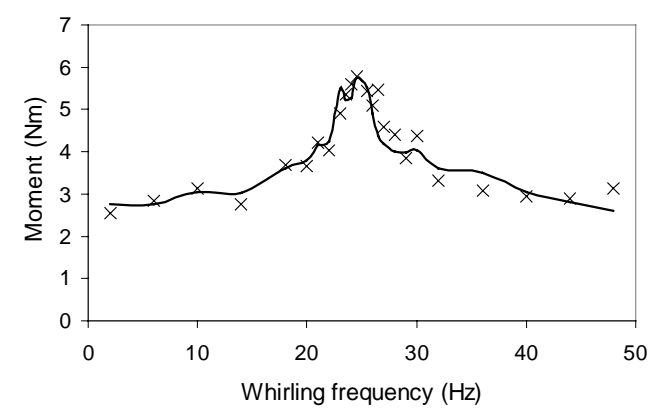

c)

Fig. 6. a) Radial force; b) Tangential force; c) Moment about the center point of the rotor as a function of whirling frequency:

- sum of the results for cylindrical and symmetric conical motion

$\times$ results of combined conical motion

Getting reliable results from the measurement of the force in combined motion at whirling frequency $f_{\mathrm{w}}=25 \mathrm{~Hz}$ at which the force reaches its maximum seemed to be impossible. The reason may be that the forces were big enough to cause bending of the shaft. For the combined motions, we did not measure the force at that whirling frequency.

Near to the synchronous whirling frequency $\left(f_{\mathrm{w}}=25 \mathrm{~Hz}\right)$, the measured moment reaches its maximum. At the other frequencies, the measured moment is notably smaller and seems to have a bit the same frequency dependence as the radial force in cylindrical motion. The explanation for this behavior may be the interbar currents. Since the test motor has a cast aluminum cage rotor, the resistance between bars through the rotor sheet stack is not infinite, and equalizing currents can flow in the end parts of the rotor cage through the rotor stack from bar to bar. This may explain the effects seen in Fig. $6 \mathrm{c}$ ).

Other kinds of combined motion was also measured, in which the frequency of the cylindrical or conical motion was kept constant (static displacement), and the forces of the other component of the motion were measured as a function of the whirling frequency.

Three cases were measured in which the rotor was performing a symmetrical conical motion (with radius $20 \mu \mathrm{m}$ ) around an axis that has a constant static displacement $(\delta)$ of, respectively, $0 \mu \mathrm{m}, 20 \mu \mathrm{m}$ and $40 \mu \mathrm{m}$ from the stator axis (Fig. 7). The moment applied in the center of the rotor is independent of the static displacement. The net force acting between the rotor and stator was almost zero at the whirling frequency range $2-48 \mathrm{~Hz}$. Fig. 8 shows the measured moments $M$ for these measurements.

In Fig. 9, the rotor is moving in cylindrical whirling motion (with radius $20 \mu \mathrm{m}$ ) around an axis that has, with respect to the stator axis, a symmetrical static conical displacement with radius $20 \mu \mathrm{m}$ at both the ends of the rotor. In Fig. 10, the forces acting between the rotor and the stator during this kind of movement are compared with the ones during a cylindrical whirling motion with same radius around the stator axis. The moment acting on the center point of the rotor is almost zero at the studied $2-48 \mathrm{~Hz}$ whirling frequencies.

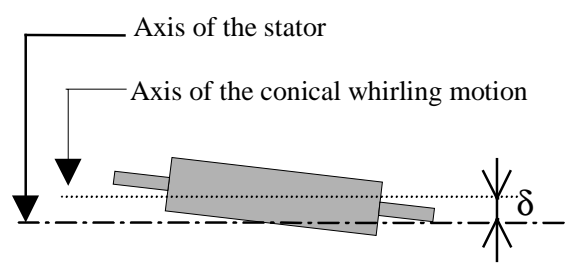

Fig. 7. Conical motion around an axis with $\delta$ static constant displacement.

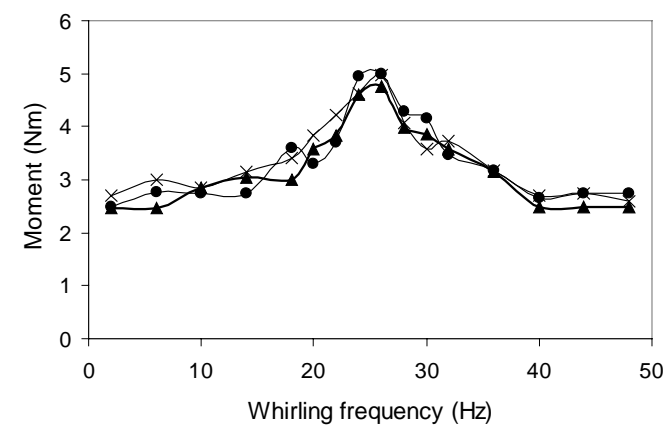

Fig. 8. Moment acting on the center point of the rotor as a function of whirling frequency for the conical motion showed in Fig. 7.

$$
\begin{aligned}
\bullet \quad \delta & =0 \mu \mathrm{m} \\
\times \quad \delta & =20 \mu \mathrm{m} \\
\Delta \quad \delta & =40 \mu \mathrm{m}
\end{aligned}
$$

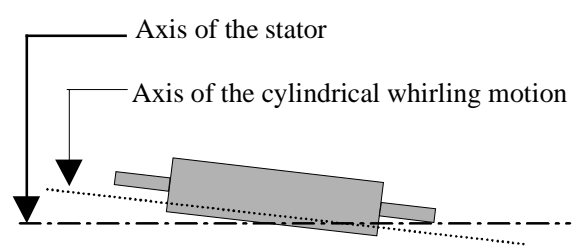

Fig. 9. Cylindrical whirling motion around an axis with symmetric conical displacement from the stator's axis. 


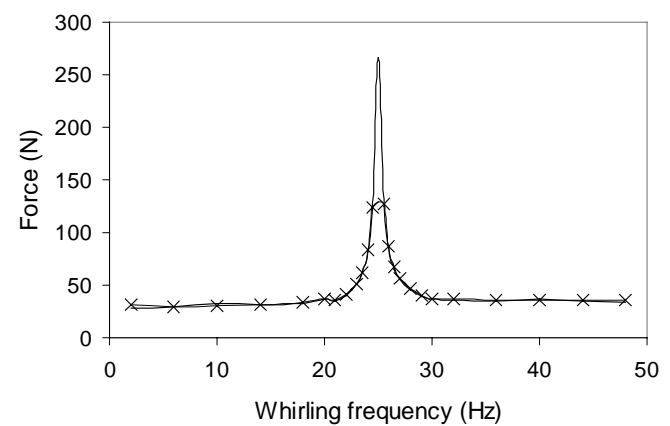

a)

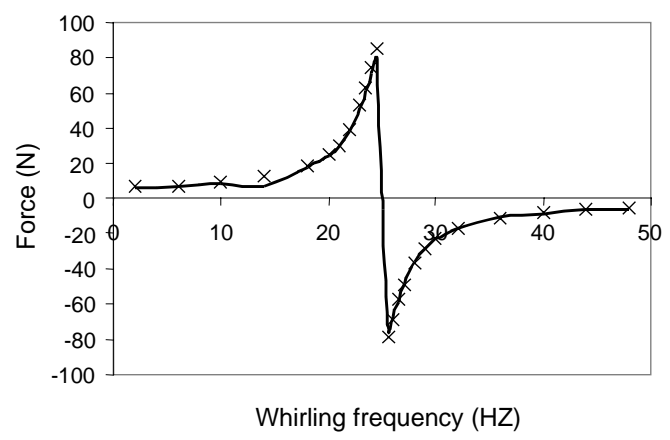

b)

Fig. 10. a) radial and b) tangential component of the force as a function of whirling frequency.

- cylindrical whirling motion around the stator's axis

$x$ cylindrical whirling motion around the axis with symmetric conical displacement

Finally, the rotor was forced to move in cylindrical whirling motion (again with radius $20 \mu \mathrm{m}$ ) around an axis that had a constant static displacement $\delta=20 \mu \mathrm{m}$ from the stator's axis shown in Fig. 11. The results for the forces acting on the rotor are shown in Fig. 12, where they are again compared with the ones measured during cylindrical whirling motion around the stator axis. Also in this case, the moment acting on the center point of the rotor is almost zero. The net force at whirling frequency $0 \mathrm{~Hz}$ was not measured, but in this case both the components of the eccentric motion would have been static displacements.

The measured results shown in Fig. 8, 10 and 12 indicate that the forces and moments caused by different whirling motions are independent of each other. The possible coupling, for example via saturation, is very small and cannot be found from the measured results.

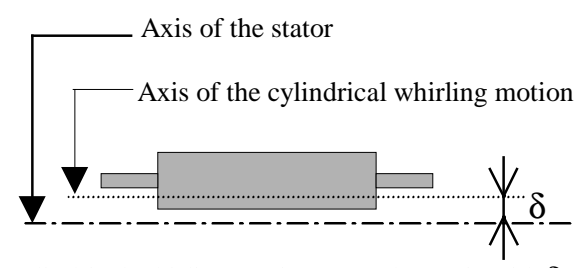

Fig. 11. Cylindrical whirling motion around an axis with $\delta$ static constant displacement.

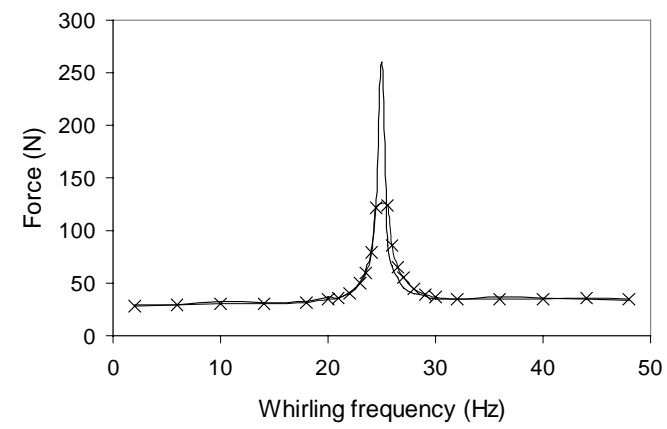

a)

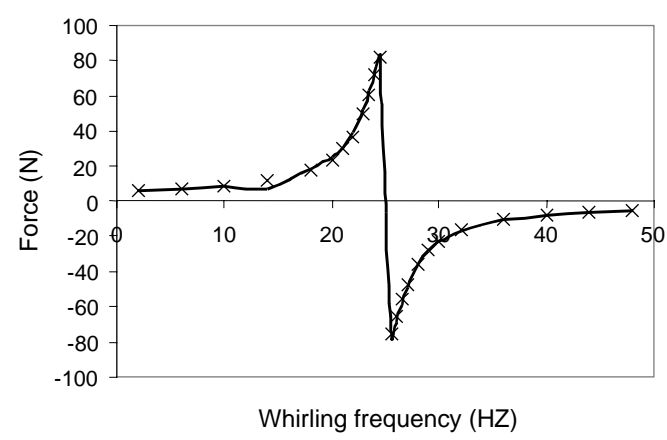

b)

Fig. 12. a) radial and b) tangential component of the force as a function of whirling frequency.

- cylindrical whirling motion around the stator's axis

$x$ cylindrical whirling motion around the axis with constant displacement

\section{DISCUSSION}

In this study we have focused on the induction motor at noload only. The reason is the used measurement set-up. The loading may influence on the measured results. Also the reduced voltage level was used to keep the magnetic bearings at the linear range of operation to guarantee the accuracy of the measurement.

The calculated and measured net forces have very good agreement but the calculated and measured moments differ notably. The reason may be the interbar currents. The test motor has a die cast aluminum cage rotor. The resistance between bars through the rotor sheet stack is not infinite and equalizing currents can flow from bar to bar through the rotor stack. Another reason may be an inaccuracy on the measurements. The accuracy of the used measurement suffers if the measured forces acting on the magnetic bearing are very small, which was the case when measuring the forces in conical motions. One possible source of error is the measurement itself. Maybe, the rotor and the stator are not perfectly cylindrical. Then the defined rotor trajectory in the calibration can neither be perfect.

Anyway, both the calculated and measured results show that the combined forces of the different whirling motions are equal with the forces of the combined motions. This conclusion is already quite straightforward from the assumption of the spatial linearity. In this study, we have not investigated 
when the assumption of spatial linearity is valid, but, based on the literature $[4,5,11]$, one can assume that spatial linearity is valid for small rotor displacements. If the point of interest is mechanical vibration of the rotor and electromechanical interaction, in which the amplitudes of the rotor vibrations are usually very small, compared with the air gap length, the spatial linearity is valid. The rotor vibrations can be presented by a sum of whirling motions, which can be handled separately.

\section{CONCLUSION}

The electromagnetic force acting between the rotor and stator, when the rigid rotor is performing eccentric motions, is studied. The magnetic field of the induction motor was solved using multi-slice, time-stepping finite element analysis and the forces were computed from the air gap magnetic field. An induction motor was equipped with active magnetic bearings for validating the calculated forces. The active magnetic bearings were used to create the eccentric motion of the rotor and also to measure the forces. The measured and calculated results have quite a good agreement. The results show that the forces of the cylindrical and the symmetric conical motion can be combined and the result is the force in the combined cylindrical and symmetric conical motion of the rotor.

\section{REFERENCES}

[1] W. Freise, and H. Jordan, "Einseitige magnetische Zugkräfte in Drehstrommaschinen", ETZ-A, vol. 83, no. 9, 1962, pp. 299-303.
[2] R. Belmans, A. Vandenput, and W. Geysen, "Calculation of the flux density and the unbalanced pull in two pole induction machines", $\mathrm{Ar}$ chiv für Elektrotechnik, vol. 70, 1987, pp. 151-161.

[3] A. C. Smith, and D. G. Dorrell, "Calculation and measurement of unbalanced magnetic pull in cage induction motors with eccentric rotors. Part 1: Analytical model”, IEE Proc. Electr. Power Appl., vol. 143, no. 3, 1996, pp. 193-201.

[4] J. Früchtenicht, H. Jordan, and H. O. Seinch, "Exzentnzitätsfelder als Urache von Laufinstabilitäten bei Asynchronmachinen, Parts 1 and 2", Arch. Electrotech (Germany), vol. 65, 1982, pp. 271-292.

[5] A. Arkkio, M. Antila, K. Pokki, A Simon, and E. Lantto, "Electromagnetic force on a whirling cage rotor", IEE Proc. -Electr. Power Appl., vol. 147, no. 5, 2000, pp. 353-360.

[6] A. Tenhunen, T. P. Holopainen and A. Arkkio, "Impulse method to calculate the frequency response of the electromagnetic forces on whirling cage rotors", IEE Proc. Electr. Power Appl., in press.

[7] D. Dorrell, "Modelling of non-uniform rotor eccentricity and calculation of unbalanced magnetic pull in a 3-phase cage induction motors", Proceedings of ICEM 2000, August 2000, Espoo, Finland, pp. 18201824.

[8] A. Tenhunen, "Finite-element calculation of unbalanced magnetic pull and circulating current between parallel windings in induction motor with non-uniform eccentric rotor", Proceedings of Electromotion'01, Bologna, Italy, 19-20 June 2001, pp. 19-24.

[9] A. Tenhunen and A. Arkkio, "Modelling of induction machines with skewed rotor slots", IEE Proc. Electr. Power Appl., Vol. 148, No. 1, January 2001, pp. 45-50.

[10] J. L. Coulomb, "A methodology for the determination of global electro-mechanical quantities from a finite element analysis and its application to the evaluation of magnetic forces, torques, and stiffness", IEEE Trans. on Magn., vol. 19, no. 6, 1983, pp. 2514-2519.

[11] A. Tenhunen T. P. Holopainen and A. Arkkio, "Spatial linearity of unbalanced magnetic pull in induction motors during eccentric rotor motions", 15th International Conference on Electrical Machines (ICEM 2002). 25 - 28.8.2002. Bruges, Belgium, paper number 115, 6 pages. 